\title{
Augmenting Surveillance to Minimize the Burden of Norovirus-Like IIIness in Ontario: Using TeleHealth Ontario Data to Detect the Onset of Community Activity
}

\author{
Stephanie L. Hughes* and Andrew Papadopoulos \\ University of Guelph, Guelph, ON, Canada
}

\begin{abstract}
Introduction
Norovirus, commonly referred to as the winter vomiting disease, is the most common cause of gastroenteritis worldwide, with the total number of cases reported per year in Ontario second only to the common cold. The disease is highly infectious, requires a low infectious dose, and is well-known to cause large outbreaks in closely confined populations. While deaths are rare, hospitalization and longterm sequelae are more likely to occur in at-risk populations, such as the elderly or immunocompromised. Action to reduce the number of norovirus infections per year is required due to its health and economic burden. It is estimated that norovirus infections cost the United States 2.5 billion CAD and the United Kingdom close to 200 million CAD per year in health care costs alone. While laboratory surveillance is practiced in Ontario to detect norovirus outbreaks, early detection remains a challenge. This project aims to utilize syndromic surveillance with TeleHealth Ontario data in order to develop an early warning system mitigating the impact of norovirus outbreaks.
\end{abstract}

\section{Methods}

TeleHealth Ontario call data, using vomiting as the predominant symptom for calling, will be obtained for September 2009 - August 2015. Call volumes preceding the winter vomiting season will be analysed such that "normal" call proportions (vomiting calls/total calls) can be determined, and a threshold can be identified. An alarm will be triggered when the proportion of calls with vomiting as a predominant symptom surpasses this threshold for two consecutive weeks, thus signifying the start of the norovirus winter vomiting season, typically October - April. Norovirus laboratory reports in Ontario for the same time period will be used as a comparison to identify the confirmed norovirus season.

\section{Results}

From this work, an early warning system will be created to detect norovirus outbreaks earlier than the conventional laboratoryconfirmed surveillance methods, as vomiting calls to TeleHealth Ontario will precede provincial norovirus laboratory reports. In creating such a system, public health agencies can notify hospitals, long-term care homes, and other vulnerable populations of impending outbreaks.

\section{Keywords}

Norovirus; Surveillance; TeleHealth

\section{Acknowledgments}

I would like to acknowledge the kindness from Public Health England, Public Health Ontario, Kingston, Frontenac and Lennox \& Addington Public Health, and Sykes Assistance Services Corporation in the development and execution of my project.

\section{*Stephanie L. Hughes}

E-mail: shughes@uoguelph.ca 